\title{
Une approche langagière et interactionnelle de l'argumentation
}

A Discursive and Interactional Approach to Argumentation

\section{Marianne Doury and Christian Plantin}

\section{(2) OpenEdition \\ 1 Journals}

Electronic version

URL: http://journals.openedition.org/aad/2006

DOI: 10.4000/aad.2006

ISSN: 1565-8961

Publisher

Université de Tel-Aviv

\section{Electronic reference}

Marianne Doury and Christian Plantin, « Une approche langagière et interactionnelle de I'argumentation », Argumentation et Analyse du Discours [Online], 15 | 2015, Online since 15 October 2015, connection on 23 September 2019. URL : http://journals.openedition.org/aad/2006 ; DOI : $10.4000 /$ aad.2006

This text was automatically generated on 23 September 2019.

\section{c) (†) $\Theta$}

Argumentation \& analyse du discours est mis à disposition selon les termes de la licence Creative Commons Attribution - Pas d'Utilisation Commerciale - Pas de Modification 4.0 International. 


\title{
Une approche langagière et interactionnelle de l'argumentation
}

\author{
A Discursive and Interactional Approach to Argumentation \\ Marianne Doury and Christian Plantin
}

\section{Introduction}

Les études d'argumentation reposent sur une triple tradition de recherche rhétorique, logique et dialectique - dont les œuvres d'Aristote et de Cicéron ont posé les fondements. La tradition rhétorique de l'argumentation est particulièrement présente dans les études de rhétorique aux États-Unis ${ }^{1}$, et dans la «Nouvelle Rhétorique» de Perelman et Olbrechts-Tyteca (1958). La pensée logique de l'argumentation, développée dans tous les traités de logique traditionnelle, a été mise en crise par la formalisation de la logique à la fin du 19e siècle (Frege [1879], Auroux 1995, Plantin 2005). La «Logique substantielle» de Toulmin (1958) et la «Logique Informelle " (Blair et Johnson 1980, Walton 1996) proposent des approches de l'argumentation comme étude des formes de raisonnement, reconnaissant la validité conditionnelle d'enchaînements autres que déductifs et inductifs. La pragmadialectique (van Eemeren et Grootendorst 2004) a développé une théorie générale de l'argumentation comme dialogue réglé orienté vers l'élimination des différences d'opinion, ce qui est un des usages essentiels de l'argumentation. Ces master theories de l'argumentation sont les références constitutives du champ contemporain des argumentation studies. Elles sont engagées dans un dialogue critique constant et fructueux, et leurs expériences et leurs acquis sont incontournables pour toute réflexion sur l'argumentation.

Deux autres théories de l'argumentation apparaissent comme particulièrement originales, utiles et stimulantes, la théorie de "L'Argumentation dans la langue " d'Anscombre et Ducrot (1983) et la «Logique naturelle » de Grize (1990). La première met l'accent sur les questions sémantiques, en langue; elle caractérise le sens de tout énoncé par son orientation argumentative, c'est-à-dire la classe d'énoncés qu'elle 
admet comme suite dans un discours grammaticalement bien formé. La seconde focalise sur les aspects cognitifs de l'argumentation, assimilée au mode de schématisation des objets opéré par le discours. Par rapport aux précédentes, elles présentent ainsi la particularité d'être des théories généralisées de l'argumentation (Plantin, à paraître, art. « Orientation »; « Schématisation »). Ces théories ont construit des concepts qui nous sont indispensables, comme la notion d'orientation linguistique pour la théorie de l'argumentation dans la langue, ou la notion d'objet de discours pour la logique naturelle.

Nous n'entrerons pas ici dans la course stérile aux fondamentalismes pour tenter de hiérarchiser ces théories, partant de réduire telle(s) ou telle(s) approche(s) à telle(s) ou telle(s) autre(s). Toutes nous sont précieuses, et nous nous estimons heureux de collaborer à une discipline qui admet la pluralité théorique, même si cette pluralité a pour prix une (apparente ?) dispersion des notions et des méthodes.

Dans le présent article, nous parlons d'argumentation par le langage pour souligner que nous prenons en considération les problématiques sémantiques, relevant de la langue, comme les problématiques séquentielles relevant de l'analyse du discours et des interactions. Nous nous proposons de montrer comment certaines des voies de réflexion explorées par ces approches théoriques permettent d'éclairer des données argumentatives - ce qui en constitue, à nos yeux, la raison d'être. Réciproquement, nous pensons que c'est en «faisant travailler » ces théories sur des données qu'on a la meilleure chance d'en proposer une articulation.

On partira ainsi d'une étude de cas portant sur des soirées électorales télévisées (au soir du premier tour des élections présidentielles françaises de $2012^{2}$, et au soir des élections européennes de 2014)33. On observera comment, à partir de données chiffrées, de «faits bruts", les différents intervenants construisent discursivement les résultats obtenus afin de les orienter vers une conclusion évaluative spécifique ( « c'est un bon score» / "c'est un mauvais score »). Il s'agira de montrer que, à l'opposé d'une approche considérant le langage comme un accessoire de l'argumentation certes indispensable à sa communication, mais peu digne d'intérêt, c'est bien leur mise en discours qui construit l'orientation argumentative des « faits » et prépare les énoncés à servir d'arguments pour des conclusions déterminées. En nous adossant à cette analyse, nous expliciterons ensuite les options théoriques et méthodologiques qui ont assuré notre démarche, et que nous caractériserons comme une approche compréhensive de l'argumentation ${ }^{5}$.

\section{1. Étude de cas : La construction argumentative des scores électoraux}

Quiconque a été exposé à une soirée électorale d'envergure ne peut manquer d'avoir été frappé par une évidence : alors que par définition, le vote a déjà eu lieu - et que, dans une certaine mesure, on peut considérer que «les jeux sont faits »-, journalistes et politiques se livrent à un travail effréné de construction discursive des résultats, afin de leur conférer une orientation argumentative conforme à leurs intérêts pour les seconds, à leur interprétation du scrutin pour les premiers. 


\subsection{La mise en mots}

\subsubsection{Les opérateurs argumentatifs}

Un premier travail visant à construire une orientation argumentative des résultats (favorisant des conclusions du type « c'est un bon score » ou " c'est un mauvais score ») repose sur leur mise en mots. En particulier, l'utilisation de ce que Ducrot (1983) appelle des « opérateurs argumentatifs » gouverne l'activation de certains topoï plutôt que d'autres, définissant à leur suite des enchaînements préférés.

Ainsi, lorsque Ségolène Royal, commentant le score obtenu par Nicolas Sarkozy au premier tour des élections présidentielles de 2012, évoque :

monsieur Sarkozy/ qui ne recueille que vingt-cinq pour cent des voix $/{ }^{6}$

" ne ... que ", pour reprendre les termes de Ducrot, inscrit $25 \%$ dans une série de scores décroissants, ou " oriente vers le moins » (invite à tirer des $25 \%$ obtenus par Nicolas Sarkozy les mêmes conclusions que celles qu'aurait autorisées un score inférieur) ${ }^{7}$. La présentation faite par Ségolène Royal du résultat de Nicolas Sarkozy prépare donc le terrain, argumente linguistiquement ${ }^{8}$, pour une évaluation négative de son score (en particulier, elle justifie l'emploi de la désignation de "désaveu » ou de "sanction", récurrente dans la bouche des adversaires de Nicolas Sarkozy).

\subsubsection{Pourcentages / valeurs absolues}

Un autre procédé récurrent de présentation linguistiquement orientée de scores électoraux consiste dans le passage des pourcentages aux valeurs absolues. Ce procédé oriente le résultat "vers le plus ", dans la mesure où il substitue un "grand chiffre " (de l'ordre, au minimum, du million) à un " petit » chiffre (de l'ordre de l'unité ou de la dizaine), et qu'il concrétise le score par l'évocation des électeurs, dont, quel que soit le nombre, il est difficile pour un politique en démocratie d'ignorer publiquement le vote. On voit bien l'intérêt que ce procédé peut présenter en particulier pour les candidats ayant réalisé de "petits» scores. Ainsi, lors des présidentielles de 2012, Eva Joly, créditée de $2 \%$ en début de soirée sur France 2 et appelée avec le plus grand mépris "Madame deux pour cent » par Gilbert Collard sur la même chaîne, commence son intervention ainsi :

je tiens à remercier avec chaleur/ (.) le million de Françaises et de Français/ (.) qui m'ont apporté leurs suffrages/ (.) et ce faisant (.) ont défendu (.) par leur vote (.)

l'écologie (.) l'Europe (.) et la République (.) exemplaire

On notera un arrondissement stratégique du chiffre ainsi invoqué, puisque $2 \%$ des voix correspondent en réalité à environ 720000 électeurs - ce qui constitue une approximation non négligeable. Cette approximation s'explique par le fait que le «million » constitue un seuil qu'il faut franchir pour signifier « beaucoup » : les scores inférieurs au million ne sont jamais, au cours de ces soirées, exprimés en valeur absolue.

\subsubsection{Présentation ordinale / présentation cardinale}

Enfin, le choix entre une présentation cardinale (ou numérale) des résultats (par exemple, "François Hollande est crédité de 28,3 pour cent des voix») et une présentation ordinale ( François Hollande arrive en tête ») reflète un travail similaire d'orientation argumentative des résultats. En témoigne le fait qu'en 2012, le score 
obtenu par François Hollande n'est jamais rappelé par ses partisans, qui se focalisent sur l'ordre d'arrivée des candidats, insistant le plus souvent sur celui qui «arrive en tête» :

«c'est le candidat socialiste qui arrive euh: (.) en tête/ [...] il est en tête (.) de ce premier tour » (Ségolène Royal) / "ils ont placé François Hollande\ (.) en tête» (Lionel Jospin) / «ils ont mis François Hollande en tête» (Jean-Marc Ayrault) / « François Hollande / (.) EST en tête » (Manuel Valls) / «François Hollande (.) qui est le mieux placé/ 》 (Laurent Fabius)

Ce choix d'une présentation ordinale est particulièrement frappant dans les commentaires des résultats des élections européennes de 2014. Ce qui constitue le " séisme », le « choc », le " raz-de-marée », la " gifle »- pour reprendre les désignations récurrentes -, c'est la première place obtenue par le Front National, plus encore que le score (25\%) qu'il réalise, ce qui amène Roger Karoutchi (UMP) à regretter que son parti n'ait pas fait liste commune avec les centristes :

m'enfin/ (.) globalement si on regarde/ (.) si on avait fait liste commu/ne on s'rait aux alentours de trente trente et un pour cent/ (.) malheureusement/ (.) et on s'rait/

d'vant le $F N \backslash($.$) la symbolique est forte \backslash($.$) on aurait été devant le Front National \backslash$

Le commentaire de Karoutchi illustre l'intérêt des raisonnements contre-factuels, qui permettent d'ouvrir sur un monde possible plus acceptable face à une réalité jugée insoutenable (ici, d'envisager un monde où l'UMP serait « devant le FN»).

\subsection{La mise en arguments}

Au-delà de ces mises en mots des scores obtenus par les différents candidats, qui les orientent argumentativement vers une évaluation positive ou négative, cette dernière peut faire l'objet d'une argumentation rhétorique - c'est-à-dire être intégrée, au titre de conclusion, dans une séquence argumentative plus vaste, son acceptabilité étant appuyée à une ou plusieurs prémisses.

Le procédé argumentatif le plus massivement utilisé pour justifier discursivement les évaluations des scores réalisés par les candidats est le recours à la comparaison. Le mécanisme principal de telles comparaisons est le suivant : « S1 est un bon score si S1 > S2 », ou « S1 est un mauvais score si S1 < S2 ». L'argument comparatif s'appuie sur une échelle bidirectionnelle ${ }^{9}$, sur laquelle sont distribués les scores, potentiels arguments pour les conclusions « être un bon score » ou « être un mauvais score» : 


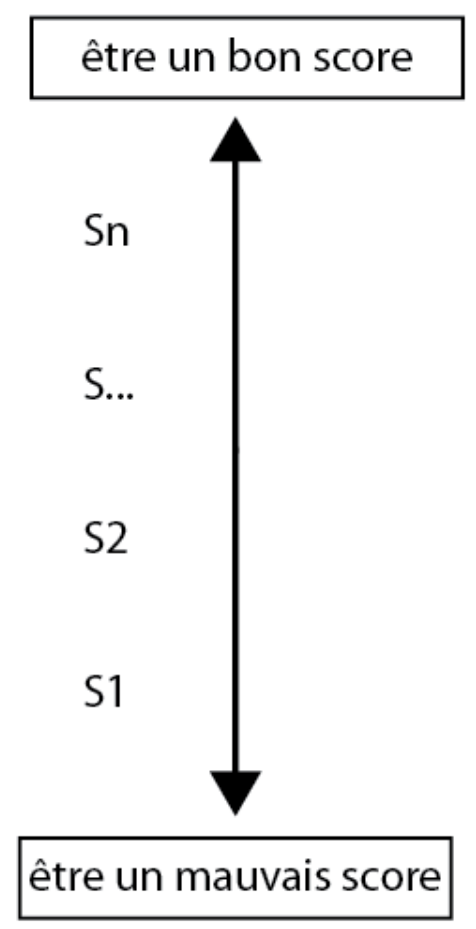

Figure 1 : Distribution des scores sur une échelle argumentative

Ainsi, lors des élections européennes de 2014 et avant même la proclamation des résultats, Michaël Szames, envoyé spécial de LCP / Public Sénat au QG du PS, pose les bases de l'évaluation du score à venir au regard d'abord des $16 \%$ obtenus par le parti lors des élections de 2009, puis du score de 1994 :

alors voilà les TROIS scénarios qui sont échafaudés ici au parti socialiste \à seize pour cent/ ce serait/ (.) quasiment une victoire/ le mê/me score qu'en 2009/ (.) ce serait (.) presque (.) INespéré pour le parti socialiste $\backslash(.$.$) en-d'ssous d'seize pour$ cent/ on va di/re (.) c'est presque normal $\backslash$ et compte-tenu des difficultés actuelles du gouvernement/ ça s'rait (.) quasiment/ aussi une victoi/re (.) même si elle s'rait amoindrie et en d'ssous/ (.) en d'ssous si jamais/ (.) ça rejoint le score de Michel Rocard en 94/ alors là/ (.) y aurait de SERieuses questions à se poser au parti socialiste

Le score pris comme point de référence pour la comparaison (le phore) est d'abord le résultat obtenu par le Parti Socialiste aux élections européennes de 2009. Szames spécifie les évaluations du score à venir au regard de sa position par rapport à ce phore : une équivalence des scores serait un argument pour conclure à une « victoire » « quasi inespérée ", un résultat en-dessous du phore (mais pas trop loin) soutiendrait une évaluation en termes de "quasi-victoire»; Szames substitue ensuite au résultat de 2009 un nouveau phore (le score obtenu par Michel Rocard, tête de liste socialiste en 1994, à savoir 14,9\%) dont la proximité serait cette fois un argument pour une évaluation négative («y aurait de sérieuses questions à se poser »).

Les comparaisons ne sont jamais définitivement concluantes, puisqu'on peut toujours leur opposer une autre comparaison qui mènerait, elle, à une conclusion différente (Govier 2001: 375). C'est cette versatilité de la comparaison quantitative, dont l'orientation argumentative varie avec la position du comparant sur l'axe considéré, qui 
en fait une ressource précieuse, toujours susceptible de servir la conclusion désirée pour peu qu'elle s'appuie sur un phore judicieusement positionné. Or, lors de soirées électorales, l'éventail des phores disponibles est large, des scores obtenus par les autres candidats à la même élection aux scores obtenus par les candidats du même bord lors d'élections antérieures, en passant par le score obtenu lors des élections précédentes par le candidat sortant, sans oublier la luxuriante ressource offerte par les prévisions des divers instituts de sondage tout au long de la campagne qui a précédé les élections. Ainsi, lors des élections présidentielles de 2012, l'invocation des sondages est massivement le fait des partisans de Nicolas Sarkozy, qui soulignent que le score obtenu par leur candidat est supérieur à celui qu'on lui avait prédit :

Xavier Bertrand : le score (.) est un score qui est Soli/de (.) et et d'ailleurs on l'voit bien/ (.) beaucoup pensaient que c'était déjà joué/ rapp'lez-vous voilà quelques s'maines (.) i(l) s'rait même pas qualifié au s'cond tour paraitt-il (.) i(l) s'rait MÊME pas (.) dans le duo de tête (.) et là aujourd'hui/ (.) j'ai ju/ste de la mémoire\

Les mêmes rappellent qu'à l'inverse, Jean-Luc Mélenchon n'a pas obtenu le « résultat mirifique » qui était annoncé :

Alain Juppé: moi je dirais que [...] l'élection ne ressemble pas à ce qu'on nous avait annoncé $[$ [...] le score mirifique annoncé à monsieur Mélenchon/ (.) n'a pas été au rendezvous/

La mise en regard des scores obtenus avec les scores annoncés obéit à la même logique que les autres formes de comparaison décrites précédemment. Le score de Jean-Luc Mélenchon, inférieur à ce qu'annonçaient les sondages, peut de ce fait être considéré comme un mauvais score ; celui de Nicolas Sarkozy, supérieur à ce qui était annoncé, est, sous cet angle, un bon score.

L'examen de ces comparaisons de scores dans un contexte interactionnel permet d'observer les objections ou réfutations auxquelles elles se heurtent, et de dégager ainsi quelques éléments d'une critique ordinaire de l'argumentation comparative ${ }^{10}$.

- Une première réaction consiste à contester la vérité des prémisses, et en particulier, à contester le score utilisé comme phore.

- Une autre réaction consiste à suggérer que la différence entre les scores comparés n'est pas suffisamment significative pour fonder une évaluation de l'un d'entre eux.

- Il est possible de mettre en cause la comparaison au motif qu'elle négligerait des différences importantes entre les deux situations comparées (on comparerait ainsi un président sortant à un ancien premier ministre, un score obtenu dans une période de crise économique à un score obtenu dans une période plus favorable, etc.).

- Une réaction courante consiste encore à proposer une autre comparaison anti-orientée, et présentée comme plus conclusive.

- Enfin, on peut suggérer qu'une argumentation comparative ne rend pas justice aux enjeux réels de la situation considérée (l'enjeu n'est pas qu'un candidat fasse mieux que le score antérieur obtenu par un autre candidat de son parti, mais que le parti lui-même obtienne un bon score - qu'il devra aux efforts cumulés des candidats successifs).

On remarquera que certaines de ces réfutations font écho aux questions critiques associées à l'argumentation comparative par les théories normatives de l'argumentation (Brown 1989, Eemeren et Grootendorst 1992 : 101-102, Whaley 1998, Doury 2009 , Walton 2014). 


\subsection{Une argumentation située}

Tous ces procédés, contribuant à argumenter linguistiquement ou rhétoriquement l'évaluation des scores, sont le fait de l'ensemble des locuteurs susceptibles d'intervenir dans les soirées électorales. On observe par ailleurs quelques phénomènes plus spécifiques, qui rappellent que l'analyse de l'argumentation doit nécessairement être rapportée à la situation dans laquelle elle s'ancre et qu'elle contribue à faire évoluer, aux locuteurs qui la prennent en charge (selon qu'ils sont, dans le cas qui nous occupe, journalistes ou politiques), à l'objet sur lequel elle porte (ici, à la nature même de l'élection), au moment où elle intervient (aussi bien au regard de la «petite histoire » que, parfois, de la «Grande ») - bref, l'argumentation est une activité située, et c'est en tant qu'activité située qu'il convient d'en rendre compte.

\subsubsection{Journalistes / politiques}

C'est aux comportements adoptés en propre par les instances énonciatives médiatiques (questions et commentaires des journalistes, mais aussi choix de mise en image, en particulier dans les duplex) qu'on s'arrêtera d'abord.

Ce qui frappe est le recours appuyé, de la part de ces instances, au registre émotionnel, qui n'est présent qu'en réaction à une sollicitation des journalistes, ou même bien souvent évité, par les politiques. Ce recours témoigne du fait que les médias privilégient, plus encore que les hommes et femmes politiques, l'enjeu de captation plutôt que l'enjeu de crédibilité (Charaudeau 2006: 8). La manifestation la plus spectaculaire des émotions liées aux soirées électorales, et de la signification argumentative qui leur est attachée, apparaît lors des directs avec les quartiers de campagne des différents partis. La disposition émotionnelle des militants, que les directs rendent accessible, est même un premier indice des résultats du scrutin, alors que l'annonce officielle des résultats n'est pas encore proclamée : des scènes de liesse valent pour évaluation positive du scrutin; des mines défaites signifient une évaluation négative aussi sûrement que les adjectifs " décevants ", les opérateurs " ne ... que », ou les substantifs « échec » ou « désaveu ». Cette mise en image des émotions est souvent soulignée par les commentaires :

Romain Messy (en direct du QG du Parti Socialiste) : oui tout à fait David (.) avec les militants du Parti Socialiste qui ont/ accueilli les résultats sur écran géant en direct sur France deux/ (.) avec une explosion de joie/[Présidentielles 2012]

Même commentaire de Guillaume Barret, qui, en direct du QG du Front National lors des mêmes élections, évoque « une explosion de joie ici hein lorsque les euh: résultats sur l'écran derrière nous s'sont inscrits/ (.) vous les entendez/ (.)»; et si l'émotion est souvent considérée comme un surgissement incontrôlé, dans un contexte où la présence des médias est si forte, son expression hyperbolique par les militants peut aussi être considérée comme stratégique, et son explicitation par les journalistes, comme une façon de reporter polyphoniquement l'évaluation du score qu'elle soustend.

Par ailleurs, c'est souvent en termes de réaction émotionnelle que les journalistes invitent les politiques à s'exprimer ; "vous êtes aussi attristé/ parlez franchement", demande Michel Grossiord à Bernard Debré au soir des élections européennes ; lors des présidentielles, David Pujadas interroge Manuel Valls sur «son sentiment, son impression" suite à l'annonce des résultats. Mais quelle que soit l'émotion suggérée 
par les journalistes (et quelle que soit l'évaluation du score vers laquelle elle oriente), les politiques s'efforcent de résister à cette pression vers l'émotif, sans doute jugée incompatible avec la dignité de leur fonction :

David Pujadas : Gilbert Collard ce soir/ quoi $\backslash$ (.) vous triomphez/ (.) vous exultez/ Gilbert Collard [affichant un visage impassible] : dans une démocratie/ on (.) on prend acte de ce que veut le peuple hein/ (.) et je voudrais moi d'abord dire à:: (.) l'ensemble des Françaises et des Français (.) qui constituent la tache indélébile ${ }^{11}$ (.) la tache indélébile/ (.) qu'ils ont tout mon respect et tous mes remerciements $\backslash$ [Présidentielles 2012]

On notera enfin l'utilisation, pour qualifier les scores obtenus par les candidats, d'adjectifs subjectifs affectifs, qui «énoncent, en même temps qu'une propriété de l'objet qu'ils déterminent, une réaction émotionnelle du sujet parlant face à cet objet " (Kerbrat-Orecchioni, 1980 : 84). En 2012, Fabien Namias qualifie d'« impressionnant » le score de Marine Le Pen ; mais l'adjectif affectif le plus fréquemment utilisé au cours de la soirée est l'adjectif "décevant ", et il qualifie les scores jugés en-dessous de ce qui était attendu, notamment celui de Jean-Luc Mélenchon, à qui le journaliste présent place Stalingrad demande :

monsieur Mélenchon vous faites MOINS de douze pour cent est-ce que vous êtes déçu

[Présidentielles 2012]

\subsubsection{Deux élections, deux régimes de commentaires}

Malgré la transversalité de certains procédés, que l'on retrouve d'une soirée électorale à l'autre, chacune d'entre elles est structurée par des enjeux propres, liés à la nature de l'élection (européenne / présidentielle / régionale / départementale / municipale), au type de scrutin et à l'étape considérée (premier ou second tour), et enfin - même si l'essentiel de cette étude de cas vise à « dénaturaliser » les commentaires de scores, et à les faire apparaître comme des constructions discursives et non comme des miroirs reflétant des « faits »- aux scores eux-mêmes.

Ainsi, nombre des procédés décrits précédemment font sens dans un contexte de soir de premier tour d'élections présidentielles ; c'est parce qu'ils préparent le second tour qu'il convient de brider l'éventuelle déception aussi bien qu'un triomphalisme hâtif, ou d'additionner les scores en anticipation sur de possibles alliances.

Par ailleurs, la stratégie qui consiste à présenter coûte que coûte le score obtenu comme un bon score et à rejeter les qualifications d' "échec", qui domine lors des élections présidentielles de 2012, est parfois suspendue. C'est ce que l'on observe lors des élections européennes du 25 mai 2014, en raison du choc généré par le score obtenu par le Front National ${ }^{12}$. Il n'est ainsi pas exceptionnel d'y entendre des échanges comme le suivant, dans lequel Marie-Noëlle Lienemann reconnaît quoique du bout des lèvres, par un raisonnement contre-factuel, l'échec des socialistes :

Michaël Szames: on l'imagine/ Marie-Noëlle Lienemann est-ce que c'est une sanction/ de la politique menée/ par l'actuel gouvernement de Manuel Valls \}

Marie-Noëlle Lienemann : bah (.) oui (.) à l'évidence enfin si tout allait bien/ on aurait pas fait ce score

De même, Roger Karoutchi, invité à réagir pour l'UMP, concède :

oh ben si j'vous disais qu'je chante: ce s'rait excessif/ (.) euh j'ai connu des soirs plus drô/les

Quoi qu'il en soit, et malgré l'accord sur l'évaluation négative du score, les partis ayant réalisé un score peu satisfaisant préfèrent battre leur coulpe dans un collectif des 
perdants aussi étendu que possible, d'où le succès de l'analyse reprise par l'ensemble des commentateurs en plateau, qui posent qu'aucun parti hormis le Front National ne peut se réjouir du score qu'il a obtenu ("un seul gagnant, beaucoup de perdants", suggère par exemple Guillaume Petit sur Public Sénat). Ainsi, il est de l'intérêt des socialistes, dans l'acceptation de leur échec, de s'associer aux autres partis. Dans cet esprit, David Assouline, interrogé sur l'échec du Parti Socialiste, résiste à l'évaluation négative suggérée par le journaliste :

ça reste je je répète/ l'abstention: massi/ve (.) qui a CERTainement été encore plus forte à gau/che parce que [...] la deuxième chose c'est une victoire du Front National/ (.) [...] et ça appelle à une réaction de TOUS les Républicains qui doivent comprendre (.) et de TOUT l'monde TOUT l'monde doit être devant sa responsabilité

$\mathrm{Au}$ terme de ces pistes d'analyse ouvertes par l'examen de la construction argumentative des commentaires de score, on reprendra dans ce qui suit certaines caractéristiques de l'approche de l'argumentation qui a sous-tendu cette étude de cas, et en particulier :

- l'importance accordée à la dimension langagière ;

- le rôle déterminant de la construction des données;

- le rapport entre théories, données et la notion d'application qui s'en dégage.

\section{L'argumentation par le langage}

Nous nous intéressons plus particulièrement aux questions de langage telles qu'elles sont abordées, implicitement ou explicitement, par les différentes théories de l'argumentation mentionnées en introduction, en adoptant, en fonction des cas considérés, la perspective de l'analyse du discours ou celle de l'analyse des interactions.

L'analyse classique de l'argumentation rhétorique constitue pour nous une forme d'analyse du discours (nous ne disons pas « son ancêtre »). Le discours rhétorique (ou adresse rhétorique, angl. public address) correspond au discours dans son acception traditionnelle, c'est-à-dire «ce qui, dit en public, traite d'un sujet avec une certaine méthode, et une certaine longueur" (Littré, art. Discours). Cette notion de discours comme "public address" n'a rien à voir avec le discours tel qu'il est défini par Foucault $(1969,1971)$, mais elle entre parfaitement dans le cadre de ce que nous appelons maintenant « analyse du discours » (Maingueneau 1976, 1990 ; Charaudeau et Maingueneau 2002) et nous semble dans la ligne de la vision perelmanienne de «l'objet de [la théorie de l'argumentation] " comme "étude des techniques discursives " orientées vers la persuasion (Perelman et Olbrechts-Tyteca [1958] : 5).

Nous rejoignons donc, dans le domaine francophone, les travaux d'Amossy sur «l'argumentation dans le discours» ([2000], 2008, 2012) qui posent que «l'argumentation est indissociable du fonctionnement global du discours » (Amossy 2008) et proposent conséquemment d'intégrer l'analyse de l'argumentation dans l'analyse du discours. Notre perspective se différencie cependant de l'approche de "l'argumentation dans le discours" en ce que nous ne défendons pas une approche « élargie » de l'argumentation dans le discours (intégrant, au-delà des discours à visée argumentative, les discours à dimension argumentative; cf. par exemple Amossy 2008). La généralisation de la notion d'argumentation est corrélative de celle d'orientation (Ducrot 1983), mais nous considérons que seules certaines orientations sont argumentatives (il existe par ailleurs des orientations descriptives, narratives...). Du 
fait qu'il y a effectivement une orientation, on ne peut donc pas conclure qu'il y a une argumentation.

Corrélativement, l'analyse de l'argumentation se focalise sur certains discours ou fragments de discours, dont l'unité de base est la séquence argumentative (argument(s) 閭 conclusion); sa délimitation est une tâche primordiale et difficile. La notion de type d'argument est pour nous une catégorie centrale de l'analyse de l'argumentation, dans la mesure où elle restitue la cohérence de la séquence argumentative, et éclaire la dynamique interactionnelle dans laquelle elle entre, en préfigurant les réactions critiques auxquelles elle va donner lieu.

L'adossement de l'analyse de l'argumentation à l'analyse du discours (voir Plantin, articles sur l'argumentation dans Charaudeau et Maingueneau 2002) est enrichi de références au système de la rhétorique classique, dont on trouvera un exposé dans Patillon (1990). Une série de travaux appuient leur approche sur la rhétorique classique et sur divers éléments relevant des sciences du langage et de la communication, par exemple Declercq (1993), Robrieux (1993), Plantin (1990, 2005), Breton (1996), Tutescu (2003), Danblon (2005), Micheli (2012).

Par ailleurs, la mobilisation de l'analyse des interactions pour traiter de certains échanges argumentatifs est naturelle, dans la mesure où le lien de l'argumentation au dialogue est aussi ancien que l'argumentation dialectique. Les premières études menées dans la perspective des interactions ordinaires se trouvent dans Cox et Willard (éds., 1982), Moeschler (1985), van Eemeren et al. (éds., 1987); on en trouve des prolongements dans Doury (2001), Doury et Kerbrat-Orecchioni (2011), Jacquin (2014).

L'articulation de ces deux approches de l'argumentation par l'analyse du discours et par l'analyse des interactions se fait par la notion de dialogisme. Nous adoptons une perspective bakhtinienne, qui fait $\mathrm{du}$ discours dialogique monolocuteur l'intériorisation de discours dialogaux (Bakhtine 1978).

S'il peut y avoir des divergences théoriques sur les parts et les fonctions respectives du langage et du raisonnement dans l'argumentation, on constate que les données examinées par ces théories sont des données langagières : il suffit d'ouvrir un ouvrage quelconque traitant de l'argumentation ordinaire pour voir que la théorie s'appuie sur des énoncés ou paires d'énoncés (qui peuvent avoir été produits par l'analyste ou recueillis à des fins d'analyse); des paragraphes ou des séquences textuelles (pratiquement toujours recueillies); des interactions ou séquences interactionnelles (très généralement enregistrées et transcrites). Les données recueillies sont dites authentiques au sens où elles n'ont pas été produites à des fins d'analyse, mais elles sont néanmoins choisies, découpées et rassemblées à de telles fins.

L'argumentation est donc une pratique langagière ; or langage et discours ne sont pas transparents. Parler, et tout spécialement argumenter, ce n'est pas "penser tout haut ». On n'argumente pas en 'mentalien', mais par un discours, tenu dans une langue naturelle qui structure ce discours. Les sciences de la langue et de la parole ont pour vocation de donner de cette structure une description qui la rende intelligible.

Donnons quelques exemples de cette approche :

- On représente de façon élémentaire la relation argument-conclusion comme une relation bi-propositionnelle (Bruno Gollnisch, en 2014: «le parti (.) de qui est/ au gouvernement/ (.) euh ressort à 14 pour cent/ [proposition 1] (.) c'est une véritable crise institutionnelle [proposition 2]»). Cette relation entre dans le chapitre on ne peut 
plus classique de l'étude des relations entre phrases, de la grammaire de la juxtaposition, de la coordination et de la subordination des énoncés. L'étude de la connexion argumentative est un chapitre de cette grammaire.

- La conclusion est présentée comme la suite "logique » de l'énoncé. Mais Grize comme Ducrot ont montré que la valeur argumentative d'un énoncé dépend de sa structuration interne et est liée au sens même des lexèmes qui le composent. Quand, en 2014, Michel Grossiord souligne que «le PARti socialiste est en-dessous de 15\%/ à QUATORze virgule huit pour cent/», la formulation même du score obtenu par le PS « contient » la conclusion (l'évaluation négative du résultat). Autrement dit, le discours naturel est "biaisé »; il ne respecte pas le principe logique d'indépendance de la conclusion par rapport à l'argument: l'argument contient toujours un peu la conclusion; la relation argument-conclusion relève aussi de la reformulation. C'est un fait dont il faut tenir compte lorsqu'on traite du raisonnable ou de la pétition de principe en argumentation.

- La forme de l'argumentation est rarement bi-propositionnelle; arguments et conclusions se présentent souvent sous la forme de discours développés. Les argumentations (et en particulier, les argumentations par les conséquences) prennent couramment des dimensions romanesques (même si, dans l'étude de cas abordée plus haut, le contrat médiatique impose des limites drastiques à l'ampleur des argumentations portées par les différents intervenants). Leur étude utilise les instruments de la Textlinguistik, de la grammaire de l'énonciation, ainsi que tous ceux qu'ont produits la théorie du discours et la stylistique du texte et de la parole.

- Pour pouvoir fonctionner comme argument, un énoncé doit être sinon incontestable, du moins incontesté. Or il y a une stylistique de la vérité des faits et de la sincérité des locuteurs, qui s'intéresse précisément à l'art d'impliciter et de soustraire à la contestation ; on pourrait, à partir du commentaire de Roger Karouchi reproduit plus haut, mettre au jour la stratégie qui consiste à attester de la sincérité du locuteur par la mise en scène de ce qu'il lui en coûte de reconnaître la vérité.

- Du point de vue langagier, un type d'argument est un «pattern discursif», et une argumentation particulière, une instanciation de ce pattern; ce qui, du point de vue linguistique, se comprend en termes d'énoncé générique et d'actualisation. On pourrait, à partir des argumentations comparatives visant à justifier l'évaluation des scores, dégager certaines règles de mise en discours de ce type d'argument et identifier certains des patterns associés.

- Discours et contre-discours schématisent différemment leurs objets et personnes de référence (pour les uns, Nicolas Sarkozy, en 2012, a «bien résisté » là où, pour les autres, il a été "désavoué ») et ne les lient pas de la même manière. La lexicométrie peut apporter beaucoup à l'étude de ce type de phénomène.

Des phénomènes de ce type laissent penser que les données en argumentation peuvent être analysées de façon cohérente en termes de grammaire et de stylistique du discours argumentatif. Soyons clairs : il ne s'agit pas d'adresser des sommations aux analystes de l'argumentation logiciens, philosophes, sociologues, psychologues, mais seulement de suggérer qu'avoir sous le coude une grammaire et un dictionnaire, appuyés sur une bonne philosophie du langage, ça peut parfois servir.

Nous ne sommes pas spécialistes d'épistémologie des sciences, ni même des sciences humaines, mais nous présentons, dans les paragraphes suivants, quelques réflexions 
sur la forme générale du travail que nous développons, avec ses retombées méthodologiques les plus évidentes.

\section{Données}

Notre recherche accorde une grande importance à la question des données, et aux interrogations corrélatives sur la structuration des données pertinentes pour telle recherche particulière. Elle se situe ainsi dans le courant ouvert par la linguistique de corpus en général et par les études d'interaction en particulier, telles qu'elles se sont constituées à la fin du $20^{\mathrm{e}}$ siècle. On sait que, contrairement à ce que suggère le terme, les « données » ne sont pas reçues mais construites, sur les bases de critères qui doivent être explicités. Construire et structurer, conserver, rendre accessibles ces données est une part essentielle du travail scientifique, coûteuse en temps et en argent.

\subsection{Question argumentative et hétérogénéité des données}

Les données cependant sont foisonnantes; leur abondance et leur hétérogénéité demandent que les corpus soient construits en fonction d'un principe organisateur, qui permettra de dire si, et dans quelle mesure, une donnée doit faire partie du corpus. Dans le cas de l'argumentation, nous considérons que c'est la question argumentative qui fournit le principe de pertinence réglant la constitution du corpus (dans l'étude de cas présentée plus haut, cette question est la détermination de l'évaluation des scores obtenus par les différents candidats). La forme générale des données associées à une question est un ensemble de discours soutenant les diverses réponses qui lui sont données, dans leurs systèmes d'alliances et d'opposition (Plantin, à paraître, art. Stase ; Question). Les interactions argumentatives ont ce privilège qu'elles fournissent immédiatement des données de ce type.

Il est très compliqué de constituer un corpus faisant l'inventaire des interventions argumentativement articulées à tel discours du président, ou à tel événement ayant provoqué une émotion sociale, et de reconstituer ce que Ebel et Fiala (1983 : 196) ont appelé un «réseau argumentatif» complet. Pour des raisons de commodité, on prend couramment pour objet, à l'intérieur de cet espace discursif, un sous-corpus (dans le cas présenté plus haut, les soirées électorales de TF1 et France 2 pour 2012, celle de LCP-Public Sénat pour 2014) ou une simple séquence. Mais il ne faut pas perdre de vue le fait que cette séquence fait partie d'un plus vaste corpus, dont la prise en compte peut faciliter l'analyse.

Il est bien entendu que l'argumentation relève du dialogue et du monologue, elle efface les frontières entre oral et écrit, et les mêmes questions peuvent être débattues sur les deux registres, dans des genres et des formats très différents. L'espace discursif mis en place par la question est par ailleurs totalement ouvert, multi-genres et multi-sites, multi-formats. Les types de données pertinentes pour l'étude de l'argumentation sont donc hétérogènes.

\subsection{Dimension interactionnelle / interdiscursive de l'argumentation}

Nous avons choisi d'organiser cette hétérogénéité des données argumentatives sur la base de principes interactionnels et interdiscursifs. L'interaction a d'abord la vertu de 
cadrer sous un format commun discours et contre-discours. En ce sens, elle simplifie le travail de construction des corpus, dans la mesure où tout épisode interactionnel où s'affiche un différend ratifié est un «bon objet »pour l'étude de l'argumentation, il ne reste plus qu'à en circonscrire correctement les frontières.

Les processus interactionnels sont internalisés par le discours monologal selon des mécanismes relevant du dialogisme et de la polyphonie. Réciproquement, cette argumentation des discours monologaux dialogiques peut être externalisée sous forme de dialogue. Du point de vue de l'éducation à l'argumentation, l'expérience montre que les étudiants ont beaucoup de mal à expliciter les formes de dialogue enfouies dans ces monologues.

Ce qui précède porte sur les données linéaires, telles qu'on les constitue pour l'étude d'un cas (d'une question argumentative). Des données se rattachant à des questions différentes peuvent être aussi regroupées de façon transversale, en vue de documenter et de discuter une question théorique, par exemples la structure de l'argumentation par le gaspillage, ou les modes de positionnement du discours d'opposition par rapport au discours de proposition.

\section{Théories, données, applications}

Nous voudrions dans ce qui suit tenter de préciser la vision que nous avons de ce qu'est, dans le cas de l'argumentation, le rapport d'une théorie à ses données, et ce qu'on entend par « application d'une théorie ».

\subsection{La théorie comme langage}

Les études d'argumentation se développent comme une science discursive, c'est-à-dire une science dont le discours se développe dans une langue ordinaire. Sa syntaxe est celle de la langue ordinaire, et ses concepts s'expriment par des termes dont la signification reste liée à leurs significations ordinaires.

Les concepts correspondent ainsi à des mots « enrégimentés ». Ils sont coordonnés en un langage analytique, ou "théorie ». "Coordonnés » veut dire qu'ils sont définis de façon homogène (le plus possible) dans leur substance et dans les relations qu'ils entretiennent les uns avec les autres : les définitions doivent être non contradictoires (ou du moins compatibles), non redondantes (ou du moins pas trop); enfin, leur ensemble doit être complet et assez copieux pour rendre compte (le mieux possible) d'un « petit monde » de données fondamentales.

\subsection{Interdépendance données / théories}

Par ailleurs, un langage théorique est défini en fonction des données dont on envisage de rendre compte ; ce n'est que par ellipse que l'on parle de «théorie » dans l'absolu; une théorie est toujours une "théorie de - ». Le fait d'être corrélée à des données ne rend pas une théorie « impure », mais en assied au contraire la pertinence. Une théorie est donc un outil qui permet de construire des représentations (modèles) de ses données attachées. Réciproquement, les problèmes rencontrés au cours de cette construction font évoluer les théories. 
Nous voyons ainsi la linguistique de l'argumentation comme une science naturelle, compréhensive, reposant sur l'observation. Elle suppose une éducation à l'observation des données. L'approche linguistique de l'argumentation donne des outils, qui, combinés avec d'autres outils compatibles tirés de la théorie classique de l'argumentation, permettent de construire des représentations adéquates et éclairantes de l'activité argumentative.

\subsection{Adéquation descriptive, adéquation explicative}

Une bonne théorie a deux vertus essentielles : elle doit être adéquate du point de vue descriptif et explicatif (nous utilisons et adaptons ici des termes et des idées provenant de Chomsky 1964). Ces deux formes d'adéquation caractérisent une approche compréhensive de l'argumentation.

\subsubsection{Adéquation descriptive}

Une théorie est descriptivement adéquate si les descriptions qu'elle permet de construire correspondent à l'intuition, donnent une image correcte, cohérente, des intuitions de l'argumentateur informé. Par exemple, prétendre décrire l'argumentation ordinaire sans prendre en compte la subjectivité ou l'émotion n'est pas descriptivement adéquat; parler systématiquement de fallacies of emotion ne l'est pas non plus, dans la mesure où l'émotion peut être légitime et argumentée, on le constate au premier regard jeté sur les données (Micheli 2010, Plantin 1998, 2011).

On peut renforcer la condition d'adéquation descriptive en prenant comme référence non plus les savoirs communs mais les savoirs partagés par la communauté des études d'argumentation ; elle demandera alors, par exemple que les divers types d'arguments soient correctement identifiés.

\subsubsection{Adéquation explicative}

Une théorie est adéquate du point de vue de l'explication si les descriptions qu'elle permet de construire jettent quelque lumière nouvelle sur les données, si elle améliore la compréhension du locuteur-argumentateur informé, voire du spécialiste de l'argumentation. Par exemple, la notion d'échelle argumentative proposée par la théorie de l'argumentation dans la langue (Ducrot 1980) a une valeur explicative en ce qu'elle permet d'intégrer à la théorie de l'argumentation les phénomènes de gradualité.

Si une représentation est en décalage par rapport à l'intuition informée, elle n'est pas mauvaise pour autant. Mais l'analyste doit alors passer à un autre niveau et montrer que sa représentation donne par exemple plus de cohérence à la description globale, ou qu'elle éclaire, ou révèle, des faits restés ignorés. L'adéquation explicative prime sur l'adéquation descriptive.

Cette conception générale du travail en argumentation appelle des précisions sur des oppositions courantes telles que l'opposition "théories vs pratiques", "théoriciens vs praticiens ", "approches théoriques vs approches empiriques »; ou encore ce qu'est "appliquer une théorie», ou encore "toute théorie de l'argumentation est nécessairement normative ». 


\section{Approches théoriques versus approches empiriques?}

On parle généralement d'application dès lors qu'on "fait tourner " un modèle de l'argumentation sur des données empiriques. L'étude des argumentations qui se développent autour du tracé d'une ligne TGV ou du développement d'un aéroport relève ainsi de l'application de la théorie de l'argumentation.

Un détour par les sciences du vivant permet d'explorer plus loin les rapports entre théorie, données et application. La théorie de l'évolution est définie en relation avec un ensemble de données auxquelles elle s'applique, au sens où elle vise à en rendre compte : «la diversification des formes de vie »: «Les explications scientifiques de la diversification des formes de la vie qui apparaissent dans la nature, depuis les premières formes de la vie jusqu'à la biodiversité actuelle, sont appelées "théories de l'évolution" ". ${ }^{13}$

Dans le titre suivant, on parle d'application de cette théorie, mais en un sens différent du précédent : «Peut-on appliquer la théorie de l'évolution aux sociétés? / La théorie de la sélection naturelle, qui suppose la compétition et l'analyse des contraintes du milieu, permet-elle de comprendre l'histoire humaine $?^{14}$ "

On se propose ici de faire fonctionner cette théorie sur de nouveaux objets, ne faisant pas partie de son domaine de définition originel, par exemple, les sociétés. On part d'une analogie "forme de la vie / forme de la société »; « évolution des organismes / histoire » :

L'application des thèses émises par le savant anglais aux sociétés humaines a donné naissance ces dernières décennies à une discipline, la psychologie évolutionniste, qui postule que la psychologie est, elle aussi, soumise aux mécanismes de la sélection naturelle ${ }^{15}$.

On voit que l'expression "appliquer une théorie à de nouvelles données " n'a pas le même sens selon que les données dites nouvelles relèvent ou non du domaine de définition de la théorie. Ni la psychologie, ni la sociologie ne relèvent du domaine de définition de la théorie darwinienne; si l'on échoue à montrer que "les sociétés humaines » ou " la psychologie » évoluent selon les lois de Darwin, on n'a pas réfuté la théorie darwinienne, dans la mesure où ces objets ne sont pas des « formes de vies » au sens darwinien du terme.

Par contre, si on fait fonctionner la théorie de l'évolution sur des données appartenant à la catégorie d'objets sur laquelle elle a été définie, on n'est pas du tout dans la même démarche. Par exemple, si l'on trouvait une «forme de vie» dont l'évolution est manifestement en contradiction avec les lois darwiniennes, alors il faudrait faire quelque chose. Un poppérien dirait que la théorie a été réfutée ; un pragmatique dirait qu'on peut toujours essayer de la réparer.

De notre point de vue, il est donc impossible de lâcher la théorie pour se consacrer aux données ou de se consacrer aux données en lâchant la théorie. Toute approche des données se fait en fonction d'une théorie. Si on oublie la théorie la description sera juste informe; si la théorie oublie ses données, si elle prend pour donnée l'inspiration du moment, traitée de façon allusive, la théorie est pauvre et précaire. Il ne suffit pas de négliger les données pour faire de la théorie pure. 
Nous récusons donc des oppositions comme "théories vs pratiques ", "théoriciens vs praticiens ». On peut parler d'approche théorique pour décrire une démarche qui construit et travaille ses données dans le but explicite de réformer la théorie, et d'approche empirique lorsque la démarche utilise la théorie pour construire une représentation de données. Dans les deux cas, théorie et données sont inséparables. Il n'existe pas de grande théorie qui ne nécessite d'être retravaillée à partir de données ; et si les données se laissent parfaitement subsumer sous la théorie, cela montre à la fois que la théorie est solide et que les données sont triviales.

\section{Les normes dans la représentation}

Non seulement la prise en compte de la question argumentative fournit le principe de pertinence permettant de construire un corpus, mais elle permet encore de poser la question du traitement adéquat des normes en argumentation. Pour nous, les stratégies normatives font partie des données et doivent être représentées comme telles par le modèle (Plantin 1995, 2009, Doury 2008).

Corrélativement, nous sommes en désaccord avec une idée relevant de la doxa prévalant dans les argumentation studies, selon laquelle une théorie de l'argumentation serait nécessairement normative, ou que c'est en raison de son caractère normatif qu'une approche de l'argumentation est digne d'être dite "théorie "; en d'autres termes, que la description des données devrait être orientée vers leur évaluation, et que le produit terminal de l'analyse devrait être un verdict « cette argumentation est / n'est pas valide ».

Nous suivons ici simplement la coutume linguistique: on a proposé plus haut de considérer la connexion argumentative comme un chapitre d'une grammaire des textes. Or, "grammaire" n'est pas synonyme de "grammaire normative ». Nous suivons également ainsi la voie ouverte par l'auteur de Fallacies lorsqu'il pose que

the logician ${ }^{16}$ does not stand above and outside practical argumentation or, necessarily, pass judgement on it. He is not a judge or a court of appeal, and there is no such judge or court: he is, at best, a trained advocate. It follows that it is not the logician's particular job to declare the truth of any statement or the validity of any argument (Hamblin 1970, p. 244-245).

Le fait que cette approche soit non-normative ne signifie pas qu'elle est aveugle aux normes. Notre approche prend parfaitement en compte un trait fondamental des argumentations ordinaires, le fait qu'elles soient évaluées en permanence; l'argumentation est une activité critique évaluative. Ce qui nous permet de le faire, c'est qu'une théorie de l'argumentation fondée sur la notion de question et d'articulation entre discours et contre-discours intègre, à titre de données, les évaluation produites par les acteurs de l'argumentation: on a ainsi associé à la présentation des argumentations comparatives justifiant l'évaluation des scores lors de soirées électorales, l'analyse des réfutations auxquelles elles donnent lieu, mettant par là même au jour certaines voies de la critique ordinaire de la comparaison. Argumenter, c'est s'engager dans un processus permanent d'évaluation, implicite ou explicite. Les réfutations et objections sont autant d'évaluations négatives en acte. Présenter une proposition face à une autre proposition équivaut à une évaluation négative de la proposition préexistante; les marques d'alignement accueillant une proposition sont des évaluations positives de cette proposition. L'évaluation peut être explicitée par exemple par des expressions «méta " évaluatives, comme « ça ne tient 
pas debout! sophisme! bêtise!». L'acquisition et le rodage des mécanismes et des niveaux d'évaluation sont essentiels pour l'apprentissage de l'argumentation.

Dire que l'évaluation est l'affaire des participants ne signifie pas que l'évaluation produite par tel ou tel participant est forcément valide, terminale. L'évaluation est un procès dialectique, elle peut être elle-même évaluée dès le tour de parole qui lui succède.

\section{L'argumentation est une activité située}

Enfin, l'argumentation ordinaire est une activité située (Suchman 1987). Cette notion a été introduite pour souligner l'importance du contexte où se situe l'action, mais aussi pour prendre en compte les (re)définitions permanentes du contexte en fonction de l'action en cours, et réciproquement.

Comme toutes les productions langagières ordinaires, l'argumentation est marquée par ses conditions d'énonciation et d'interaction: c'est ce dont on a cherché à rendre compte dans notre étude de cas, en évoquant quelques pistes de variations discursives induites par le statut des participants (journalistes ou politiques) ou par l'élection considérée (contraste entre les présidentielles de 2012 et les européennes de 2014).

Les dimensions intersubjective ${ }^{17}$ et interactionnelle ne peuvent être considérées comme un supplément communicationnel au discours argumentatif, marquant l'adaptation contingente d'un contenu à un contexte. Cela implique par exemple que les dimensions affectives et émotionnelles de la communication verbale doivent être prises en compte dans la théorie de l'argumentation; et si cette prise en compte de la subjectivité fait de nous des rhétoriciens, alors nous espérons que les rhétoriciens voudront bien nous admettre parmi les leurs.

Le raisonnable argumentatif est également situé, au sens où il est lié non pas à un système de règles universelles surplombant toutes les activités humaines, mais à des normes et des procédures locales, des «règles du lieu »: le raisonnable de la cour de justice n'est pas le raisonnable de l'assemblée délibérante. D'une façon générale, le raisonnable se cherche quelque part dans les méandres des règles de politesse, des maximes conversationnelles, des règlements et des coutumes d'assemblées.

En tant qu'activité située complexe, l'argumentation inclut non seulement des phénomènes rapportés à l'ethos ${ }^{18}$ et au pathos, les personnes, leurs intérêts, valeurs, motifs et émotions, mais également leurs systèmes de connaissances, aussi fragmentaires et mal articulés soient-ils, qu'on peut rapporter au logos; et cette coexistence des registres éthotique, pathétique et logique ne doit pas conduire à sousestimer la consistance du dernier. On peut en effet invalider l'activité argumentative ordinaire en la rapportant à l'idéal socratique d'un échange d'objectivités et de vérités telles qu'elles pourraient circuler dans un dialogue avec les dieux. Mais la vérité n'est pas pour autant inaccessible aux échanges ordinaires, où le prédicat «c'est vrai!»s'applique parfois de façon satisfaisante aux contributions de quelques participants. En tant qu'activité sublunaire, l'argumentation relève pleinement des arts de la preuve, telle qu'elle se construit et s'administre dans une vaste famille de sciences, allant de la physique à la médecine, l'histoire, et l'enquête judiciaire.

Les variations du domaine de savoir dans lequel s'inscrit l'argumentation entrainent des variations du langage qui l'exprime. Dans son usage ordinaire, le langage est 
systématiquement orienté, ou biaisé, ce qui ne le rend pas pour autant radicalement fallacieux. Rechercher un langage "neutre", excluant la subjectivité et l'intentionnalité, c'est s'engager sur la voie de la construction d'un ortho-langage dont le lexique et la syntaxe sont régis par les lois du calcul et de l'inférence formelle, en rupture avec le langage ordinaire. Cette démarche est possible, légitime, banale; elle est à l'œuvre dans la difficile transition du langage ordinaire aux langages de connaissance. Elle implique une rupture avec le raisonnable ordinaire de la langue et du discours, dans lequel se traite une certaine partie des affaires humaines ordinaires.

Le recours à un langage de connaissance traduit l'ambition épistémique de l'argumentation, mais celle-ci peut se réaliser sans nier pour autant la dimension intersubjective de l'argumentation. La « subjectivité épistémique » est prise en compte pour l'étude des processus guidés de construction des connaissances, en particulier dans l'éducation scientifique élémentaire. La recherche actuelle en argumentation est particulièrement active et novatrice dans le domaine de l'éducation scientifique, où l'on argumente pour apprendre et où l'on apprend à argumenter. D'une façon générale, en tant qu'activité de raisonnement, l'argumentation est fondée sur de l'information, des connaissances et des procédures structurées; dans son travail argumentatif, le locuteur peut être engagé dans un processus de construction de savoirs. Ce point nous sépare de rhétoriciens qui n'admettraient pour bases de l'argumentation que des lieux communs articulés par le sens commun.

\section{Conclusion : Une approche compréhensive de l'argumentation}

En résumé, nous approchons l'argumentation comme une activité langagière, ayant affaire à la force structurante du langage, sur laquelle elle peut s'appuyer et dont elle peut, si nécessaire, se déprendre; comme une activité intersubjective, dans toutes les dimensions de la subjectivité, y compris la subjectivité épistémique; comme une interaction critique, où les évaluateurs sont les participants. La tâche des études d'argumentation est de construire un langage analytique substantiel et cohérent, rendant possible la construction de représentations éclairantes de données construites, qui permettent de le mettre à l'épreuve.

Ces représentations peuvent, dans une certaine mesure, faire l'objet de restitution aux argumentateurs informés (adéquation descriptive); elles doivent, dans tous les cas, éclairer ces pratiques (adéquation explicative). Sur cette base, à la suite de Max Weber (1913), nous parlerons d'approche compréhensive de l'argumentation, c'est-à-dire d'une approche qui prend en compte le sens que les interlocuteurs donnent à leurs activités argumentatives, les savoirs qu'ils construisent sur ces activités, et leurs intentions, telles qu'elles s'y manifestent. En particulier, nous prenons au sérieux et reconnaissons la valeur des évaluations réciproques qu'ils portent sur leurs activités argumentatives. 


\section{BIBLIOGRAPHY}

Amossy, Ruth. 2012 [2000]. L'argumentation dans le discours (Paris : Nathan)

Amossy, Ruth. 2008. « Argumentation et analyse du discours : perspectives théoriques et découpages disciplinaires », Argumentation et Analyse du Discours 1 [En ligne : http:// aad.revues.org/200]

Amossy, Ruth. 2010. La présentation de soi. Ethos et identité verbale (Paris : Presses Universitaires de France)

Amossy, Ruth. 2012. «Faut-il intégrer l'argumentation dans l'analyse du discours? Problématiques et enjeux ", Argumentation et Analyse du Discours 9 [En ligne : http:// aad.revues.org/1346

Anscombre, Jean-Claude \& Oswald Ducrot. 1983. L'argumentation dans la langue (Liège \& Bruxelles : Mardaga)

Auroux, Sylvain. 1995. « Argumentation et anti-rhétorique. La mathématisation de la logique classique », Hermès 15, 129-144

Bakhtine, Mikhaïl M. 1978. Esthétique et théorie du roman (Paris : Gallimard)

Blair, J. Anthony \& Ralph H. Johnson. 1980. « The Recent Development of Informal Logic », J. Anthony Blair \& Ralph H. Johnson (éds.), Informal logic: The first international symposium (Inverness, CA : Edgepress), 3-28

Breton, Philippe. 1996. L'argumentation dans la communication (Paris : La Découverte)

Brown, William R. 1989. « Two Traditions of Analogy », Informal Logic $11: 3,161-172$

Charaudeau, Patrick. 2006. « Discours journalistique et positionnements énonciatifs. Frontières et dérives ", Semen 22, 29-44

Charaudeau, Patrick \& Dominique Maingueneau. 2002. Dictionnaire d'analyse du discours (Paris : Seuil)

Chomsky, Noam. 1964. Current issues in linguistic theory (The Hague : Mouton)

Cox, J. Robert \& Charles Arthur Willard (éds.). 1982. Advances in Argumentation Theory and Research (Carbondale, IL : Southern Illinois University Press)

Danblon, Emmanuelle. 2005. La fonction persuasive. Anthropologie du discours rhétorique : origines et actualité (Paris : Colin)

Declercq, Gilles. 1993. L'art d'argumenter. Structures Rhétoriques et littéraires (Paris : Éditions Universitaires)

Doury, Marianne. 2001. « Une discussion dans un commerce d'habitués », Les Carnets du Cediscor 7, 119-134

Doury, Marianne. 2008. « “Ce n'est pas un argument !” Sur quelques aspects des théorisations spontanées de l'argumentation », Pratiques 139 (« Les Linguistiques populaires, Paveau, MarieAnne \& G. Achard-Bayle, éds), 111-128

Doury, Marianne. 2009. «Argument Schemes Typologies in Practice : the Case of Comparative Arguments », Eemeren, Frans H. van \& Bart Garssen (éds), Pondering on Problems of Argumentation: Twenty Essays on Theoretical Issues (Dordrecht : Springer), 141-156 
Doury, Marianne \& Catherine Kerbrat-Orecchioni. 2011. « La place de l'accord dans l'argumentation polémique : le cas du débat Sarkozy/Royal (2007) », À contrario 16 : 2, 63-87

Doury, Marianne \& Assimakis Tseronis. 2014. «Les faits et les arguments : la mise en discours des scores électoraux », Jan Goes, Jean-Marc Mangiante, Françoise Olmo \& Carmen Pineira (éds), Le langage manipulateur. Pourquoi et comment argumenter ? (Arras : Presses Universitaires d'Artois), 193-210

Ducrot, Oswald. 1980. Les échelles argumentatives (Paris : Minuit)

Ducrot, Oswald. 1983. « Opérateurs argumentatifs et visée argumentative », Cahiers de linguistique française 5, 7-36

Ducrot, Oswald. 1995. « Les modificateurs déréalisants », Journal of Pragmatics 24 :1, 145-165

Ducrot, Oswald. 2004. « Argumentation rhétorique et argumentation linguistique », Doury, Marianne \& Sophie Moirand (éds), L'argumentation aujourd'hui. Positions théoriques en confrontation (Paris : Presses de la Sorbonne Nouvelle), 17-34

Ebel, Marianne \& Pierre Fiala. 1983. Sous le consensus, la xénophobie. Paroles, arguments, contextes (1961-1981), Thèse, institut de Science Politique, Mémoires et documents 16, Lausanne

Eemeren, Frans H. van \& Rob Grootendorst. 1992. Argumentation, Communication, and Fallacies : a Pragma-Dialectical Perspective (Hillsdale, NJ : Erlbaum)

Eemeren, Frans H. van \& Rob Grootendorst. 2004. A Systematic Theory of Argumentation: The Pragma-Dialectical Approach (Cambridge : Cambridge University Press)

Eemeren, Frans H. van, Rob Grootendorst, J. Anthony Blair \& Charles A. Willard (éds). 1987. Argumentation: Perspectives and Approaches (Dordrecht : Foris)

Foucault, Michel. 1969. L'archéologie du savoir (Paris : Gallimard)

Foucault, Michel. 1971. L'ordre du discours (Paris : Gallimard)

Frege, Gottlob 1999 [1879]. Idéographie. Traduction de l'allemand [Begriffschrift], préface, notes et index par Corine Besson. Post-face de Jonathan Barnes (Paris : Vrin)

Govier, Trudy. 2001. A practical study of argument (5th edition) (Belmont : Wadsworth)

Grize, Jean-Blaise. 1990. Logique et langage (Paris : Ophrys)

Hamblin, Charles L. 1970. Fallacies (Londres : Methuen)

Jacquin, Jérôme. 2014. Débattre. L'argumentation et l'identité au cœur d'une pratique verbale (Bruxelles : De Boeck)

Kerbrat-Orecchioni, Catherine. 1980. L'énonciation. De la subjectivité dans le langage (Paris : Armand Colin)

Maingueneau, Dominique. 1976. Initiation aux méthodes de l'analyse du discours (Paris : Hachette)

Maingueneau, Dominique. 1990. L'analyse du discours (Paris : Hachette)

Micheli, Raphaël. 2010. L'émotion argumentée. L'abolition de la peine de mort dans le débat parlementaire français (Paris : Le Cerf)

Micheli, Raphaël. 2012. «Les visées de l'argumentation et leurs corrélats langagiers : une approche discursive », Argumentation et Analyse du Discours [En ligne : http://aad.revues.org/1406] Moeschler, Jacques. 1985. Argumentation et conversation. Éléments pour une analyse pragmatique du discours (Paris : Hatier) 
Patillon, Michel. 2005 [1990]. Éléments de rhétorique classique (Paris : Armand Colin)

Plantin, Christian. 1990. Essais sur l'argumentation (Paris : Kimé)

Plantin, Christian. 1995. «L'argument du paralogisme », Hermès 15-16, 241-258

Plantin, Christian. 1998. « Les raisons des émotions », Bondi, Marina (éd.) Forms of argumentative discourse / Per un'analisi linguistica dell'argomentare (Bologne : CLUEB), 3-50

Plantin, Christian. 2005. L'argumentation. Histoire, théories, perspectives (Paris : PUF)

Plantin, Christian. 2009. "Critique de la parole : les fallacies dans le discours argumentatif », Vahram, Atayan \& Daniela Pirazzini (éds), Argumentation : théorie - langue - discours. (Frankfurt : Peter Lang), 51-70

Plantin, Christian. 2011. Les bonnes raisons des émotions. Principes et méthode pour l'étude du discours émotionné (Berne : Peter Lang)

Plantin, Christian (à paraître). Dictionnaire de l'argumentation. Une introduction notionnelle aux études d'argumentation (Lyon : ENS Éditions)

Perelman, Chaïm \& Lucie Olbrechts-Tyteca. 1988 [1958]. Traité de l'argumentation. La nouvelle rhétorique (Bruxelles : Éditions de l'Université de Bruxelles)

Robrieux, Jean-Jacques. 1993. Éléments de rhétorique et d'argumentation (Paris : Dunod)

Suchman, Lucy A. 1987. Plans and Situated Actions : the Problem of Human-Machine Communication (Cambridge : Cambridge University Press)

Toulmin, Stephen. 1958. The Uses of Argument (Cambridge : Cambridge University Press)

Tutescu, Mariana. 2003. L'argumentation. Introduction à l'étude du discours (Bucarest : Editura UniversitãŢiidin Bucureşti) [En ligne : http://ebooks.unibuc.ro/lls/MarianaTutescuArgumentation/1.htm]

Walton, Douglas. 1996. Argumentation Schemes for Presumptive Reasoning (Mahwah, NJ : Erlbaum)

Walton, Douglas. 2014. « Argumentation Schemes for Argument from Analogy », Henrique Jales Ribeiro (éd.), Systematic Approaches to Argument by Analogy (Dordrecht : Springer), 23-40

Weber, Max. 1913. « Über einige Kategorien der verstehenden Soziologie », http:// www.sociosite.net/topics/texts/weber_1913.pdf ; Max Weber, « De la sociologie compréhensive ", Les cahiers psychologie politique [En ligne : http://lodel.irevues.inist.fr/ cahierspsychologiepolitique/index.php?id=1950]

Whaley, Bob. 1998. «Evaluations of Rebuttal Analogy Users: Ethical and Competence Considerations », Argumentation $12: 3,351-365$

\section{NOTES}

1. Voir notamment le numéro 6 (2011) de la revue Argumentation et Analyse du Discours, consacré à «L'analyse rhétorique aux États-Unis. Hommage à Michael Leff ».

2. On trouvera une analyse plus détaillée des commentaires de scores lors de cette soirée électorale dans Doury et Tseronis 2014.

3. Les analyses qui suivent portent sur la transcription intégrale des soirées proposées par TF 1 et France 2 au soir du premier tour (22 avril) des élections présidentielles de 2012, et sur une transcription partielle (seules les formulations des scores ont été retenues) de la soirée électorale diffusée par LCP/Public Sénat à l'occasion des élections européennes du 25 mai 2014. Aucun 
comptage des phénomènes observés n'a été réalisé, mais nous avons considéré que l'observation systématique des formulations des scores et de leur évaluation permettait des jugements quantitatifs globaux (« rare », « fréquent », « massif »...) des procédés analysés.

4. Pour une réflexion plus large sur l'exploitation argumentative de données chiffrées, on pourra se référer à un numéro récent de la revue Mots. Les langages du politique $\left(\mathrm{n}^{\circ} 100,2012 / 3\right.$, «Chiffres et nombres dans l'argumentation politique »).

5. Nous adoptons ainsi la position exprimée par Max Weber (1913) à propos de la sociologie: « L'activité spécifiquement importante pour la sociologie consiste en particulier en un comportement qui 1) suivant le sens subjectif visé par l'agent est relatif au comportement d'autrui, qui 2) se trouve coconditionné au cours de son développement par cette relation significative [sinnhafte Bezogenheit] et qui 3) est explicable de manière compréhensible à partir de ce sens visé (subjectivement). »

L'objet de l'analyse est une séquence argumentative ( « un comportement » verbal d'un certain type), envisagé "selon le sens que lui donne l'argumentateur», ce sens étant "relatif au comportement d'autrui» (valeur fondamentalement interactionnelle de l'intervention argumentative); la séquence se développe de façon conditionnée "par cette relation significative ", et cette séquence est " explicable de manière compréhensible à partir de ce sens visé (subjectivement) »; nous prenons ici " explicable » au sens de « on peut rendre compte de », (voir ci-dessous la question de l'adéquation descriptive).

6. Dans les extraits proposés, / indique une intonation montante, \une intonation descendante ; (.) (..) (...) des pauses intra-répliques plus ou moins brèves. Les élisions sont notées par une apostrophe, les allongements, par : . Les commentaires en italiques entre crochets spécifient des comportements non-verbaux ou paraverbaux. [...] indique qu'un passage n'a pas été reproduit. Les majuscules signalent une intensité articulatoire accrue. Les italiques mettent en relief le point sur lequel porte le commentaire.

7. Ducrot a proposé plusieurs descriptions du fonctionnement de « ne ...que ...»; celle que l'on trouvera dans Ducrot 1995 (155 et ss.) s'adosse à la notion de modificateur réalisant/déréalisant, absente des descriptions antérieures (Ducrot 1983 notamment).

8. L'opposition entre argumentation linguistique et argumentation rhétorique, telle qu'elle apparaît dans cet article, est librement inspirée de Ducrot (2004). On parlera d'argumentation linguistique pour référer au fait que la façon de « dire les choses », leur mise en mots, les prépare déjà à servir certaines conclusions plutôt que d'autres. L'expression «argumentation rhétorique » renverra à l'étude des séquences argumentatives, et s'appuiera notamment sur la catégorie analytique largement partagée au sein des études en argumentation de "types d'arguments ».

9. Cette représentation s'inspire de la notion d'échelle argumentative (Ducrot 1980), adaptée à la spécificité du phénomène considéré - ici, au fait que les deux pôles de l'axe des scores («bon score » / " mauvais score ») sont mobilisés dans les jugements évaluatifs conclusifs.

10. Certaines des voies de réfutation prises isolément ne sont pas spécifiques à la discussion de la comparaison; c'est la panoplie composée de l'ensemble de ces réactions possibles qui trace les contours d'un mode de critique propre à l'argumentation comparative.

11. Collard reprend ici les propos d'Eva Joly, qui avait qualifié le score du FN de «tache indélébile sur les valeurs de la démocratie ».

12. Les premières estimations donnaient le FN à $25,0 \%$, l'UMP à $20.3 \%$, le PS-PRG à $14.7 \%$ - ce dont le FN a tiré argument pendant toute la soirée pour se présenter comme «premier parti de France ".

13. http://fr.wikipedia.org/wiki/Évolution_(biologie) - consulté le 13-03-2015.

14. http://www.scienceshumaines.com/peut-on-appliquer-la-theorie-de-l-evolution-auxsocietes_fr_1621.html-consulté le 13-03-2015. 
15. http://www.unige.ch/communication/Campus/campus95/dossier1/dossier.pdf - consulté le 13-03-2015.

16. Par « logician », il faut entendre « théoricien de l'argumentation ».

17. C'est pourquoi la notion de point de vue est inhérente à l'activité linguistique ordinaire.

18. Sur l'ethos dans l'argumentation rhétorique, voir Amossy (2010).

\section{ABSTRACTS}

This paper is based on a case study focused on election night specials (2012 French presidential elections; 2014 European Parliament elections in France). Specifically we observe how "rough facts" (the scores that are expressed by figures which are officially announced and undisputed) are discursively oriented towards a specific evaluative conclusion ("this is a good score" / "this is a bad score"). Based on this analysis we will make explicit the theoretical and methodological choices that back up our approach, which we characterize as a comprehensive approach to argumentation. This approach is founded notably on the notion of argumentative question (stasis) determining the structure of the data that are supposed to be represented (modeled); it also assumes that the evaluation of arguments falls to the arguers rather than to the analyst.

Cette contribution repose sur une étude de cas portant sur des soirées électorales télévisées (élection présidentielle française, 2012; élections européennes de 2014, en France). Nous observons notamment comment les «faits bruts» (données chiffrées officielles et non contestées) sont orientés discursivement vers une conclusion évaluative spécifique (« c'est un bon score " / "c'est un mauvais score »). En nous adossant à cette analyse, nous explicitons ensuite les options théoriques et méthodologiques qui ont assuré notre démarche, que nous caractérisons comme une approche compréhensive de l'argumentation. Elle est fondée notamment sur la notion de question argumentative déterminant la structure des données qu'il s'agit de représenter (modéliser) et la restitution de l'évaluation aux participants.

\section{INDEX}

Keywords: data, debate, figures, orientation, political argument, question, representation Mots-clés: argumentation politique, chiffres, débat, données, orientation, question, représentation

\section{AUTHORS}

\section{MARIANNE DOURY}

Laboratoire Communication et Politique - IRISSO, CNRS ; Université Paris Dauphine

\section{CHRISTIAN PLANTIN}

Laboratoire Interactions, Corpus, Apprentissages, Représentations - ICAR, CNRS ; Université Lyon 2 ; École Normale Supérieure de Lyon 